\title{
Stretching of the toroidal field and generation of magnetosonic waves in differentially rotating plasma
}

\author{
A. Bonanno ${ }^{1,2}$ and V. Urpin ${ }^{1,3}$ \\ 1 INAF, Osservatorio Astrofisico di Catania, via S. Sofia 78, 95123 Catania, Italy \\ e-mail: abo@oact.inaf.it \\ 2 INFN, Sezione di Catania, via S. Sofia 72, 95123 Catania, Italy \\ 3 A.F.Ioffe Institute of Physics and Technology and Isaac Newton Institute of Chile, Branch in St. Petersburg, 194021 St. Petersburg, \\ Russia
}

Received 26 April 2007 / Accepted 18 July 2007

\section{ABSTRACT}

\begin{abstract}
Aims. We evaluate the generation of magnetosonic waves in differentially rotating magnetized plasma.
Methods. Differential rotation leads to an increase of the azimuthal field by winding up the poloidal field lines into the toroidal field lines. An amplification of weak seed perturbations is considered in this time-dependent background state.

Results. It is shown that seed perturbations can be amplified by several orders of magnitude in a differentially rotating flow. The only necessary condition for this amplification is the presence of a non-vanishing component of the magnetic field in the direction of the angular velocity gradient.
\end{abstract}

Key words. magnetohydrodynamics (MHD) - instabilities - accretion, accretion disks - galaxies: magnetic fields stars: magnetic fields

\section{Introduction}

In astrophysical bodies, differential rotation is often associated with magnetic fields of various strength and geometry. If the poloidal field has a component parallel to the gradient of angular velocity, then differential rotation can stretch toroidal field lines from the poloidal ones. In the presence of the magnetic field, differential rotation can be the reason for various magnetohydrodynamic instabilities, particularly if the field geometry is complex. Some of these instabilities occur in the incompressible limit (Velikhov 1959; Fricke 1969; Acheson 1978; Balbus \& Hawley 1991) which applies if the magnetic field is weak and the Alfvén velocity is smaller than the sound speed. Other instabilities become important only in sufficiently strong magnetic fields when the effect of compressibility plays a significant role (Pessah \& Psaltis 2005; Bonanno \& Urpin 2006, 2007). Note that incompressible instabilities can often be suppressed by a strong magnetic field. For example, the magnetorotational instability (MRI) does not occur if the magnetic field satisfies the condition $B^{2}>-8 \pi \rho s \Omega \Omega^{\prime} L^{2}$ where $\rho$ is the density, $L$ is the lengthscale of disturbances, $\Omega=\Omega(s)$ is the angular velocity, and $s$ is the cylindrical radius; $\Omega^{\prime}=\mathrm{d} \Omega / \mathrm{d} s$ (see, e.g., Urpin 1996; Kitchatinov \& Rüdiger 1997). Moreover, if $\Omega$ increases with the cylindrical radius MRI cannot arise.

In recent years, many simulations of differentially rotating magnetized bodies have been performed, and much of the dynamics was interpreted as being a direct consequence of the MRI (Brandenburg et al. 1995; Hawley at al. 1995; Matsumoto \& Tajima 1995; Hawley 2000). Obviously, the MRI cannot be the only instability that operates in a rotating magnetized gas. For example, stratification can lead to a number of strong non-axisymmetric instabilities (Agol et al. 2001; Narayan et al. 2002; Keppens et al. 2002). Blokland et al. (2005) consider the influence of a toroidal field on the growth rate of the MRI and find that it leads to overstability (complex eigenvalue). Van der Swaluw et al. (2005) study the interplay between different instabilities and argue that the growth rate of convection can be essentially increased due to magnetorotational effects. Note, however, that these studies treat the stability of the magnetic field with a vanishing radial component, a condition which is often not met in astrophysical bodies. In fact, the presence of a radial magnetic field can change substantially the stability properties (Bonanno \& Urpin 2006, 2007).

In this paper, we consider stability of a differentially rotating gas in the presence of a non-vanishing radial magnetic field. Differential rotation causes the azimuthal field to increase with time by winding up the poloidal field lines into the toroidal ones. Therefore, a development of small perturbations occurs in the time-dependent background state. We show that stretching of the azimuthal field leads to the generation of magnetosonic waves in a flow. Magnetohydrodynamic waves and turbulence generated by this instability can play an important role in enhancing transport processes in various astrophysical bodies, such as accretion and protoplanetary disks, galaxies, stellar radiative zones, etc.

\section{Basic equations}

The equations of compressible MHD read

$$
\begin{aligned}
& \dot{\boldsymbol{v}}+(\boldsymbol{v} \cdot \nabla) \boldsymbol{v}=-\frac{\nabla p}{\rho}+\boldsymbol{g}+\frac{1}{4 \pi \rho}(\nabla \times \boldsymbol{B}) \times \boldsymbol{B}, \\
& \dot{\rho}+\nabla \cdot(\rho \boldsymbol{v})=0 \\
& \dot{\boldsymbol{B}}-\nabla \times(\boldsymbol{v} \times \boldsymbol{B})=0 \\
& \nabla \cdot \boldsymbol{B}=0 .
\end{aligned}
$$


Our notation is as follows: $\rho$ and $\boldsymbol{v}$ are the gas density and velocity, respectively; $p$ is the pressure; $\boldsymbol{B}$ is the magnetic field and $\boldsymbol{g}$ is gravity. In this paper, we consider an isothermal gas and assume

$p=c_{\mathrm{s}}^{2} \rho$,

where the sound speed, $c_{\mathrm{s}}$, is constant.

We work in cylindrical coordinates $(s, \varphi, z)$ with the unit vectors $\left(\boldsymbol{e}_{s}, \boldsymbol{e}_{\varphi}, \boldsymbol{e}_{z}\right)$. The basic state on which the stability analysis is performed is assumed to be axisymmetric with the angular velocity $\Omega=\Omega(s)$ and $\boldsymbol{B} \neq 0$. In the presence of the nonvanishing radial field $B_{s}$ and differential rotation, the azimuthal field increases with time by winding up the radial field lines. If the magnetic Reynolds number is large, then one obtains from Eq. (4) that the azimuthal field grows linearly with time in the basic state,

$B_{\varphi}(t)=B_{\varphi}(0)+s \Omega^{\prime} B_{s} t$,

where $B_{\varphi}(0)$ is the azimuthal field at $t=0$. A growth of $B_{\varphi}$ given by Eq. (6) can last only while diffusion of the toroidal field is negligible. Eventually, in the presence of a finite diffusivity, a steady state will emerge where winding up is balanced by diffusion of the azimuthal field. The time-scale to reach this steady state is approximately equal to the diffusion time-scale, $\sim s^{2} / \eta$, where $\eta$ is the magnetic diffusivity. Therefore, our consideration is valid only for $t<s^{2} / \eta$. However, for astrophysical plasmas, this time can be very long because of low $\eta$ and large length-scale.

For the sake of simplicity, we assume that gravity is radial, $\boldsymbol{g}(s)=-g(s) \boldsymbol{e}_{s}$, and the basic state is in hydrostatic equilibrium, then

$$
\frac{\nabla p}{\rho}=\boldsymbol{D}+\frac{1}{4 \pi \rho}(\nabla \times \boldsymbol{B}) \times \boldsymbol{B}, \quad \boldsymbol{D}=\boldsymbol{g}+\Omega^{2} \boldsymbol{s} .
$$

We consider the basic state in which $B_{s} \propto B_{\varphi}(0) \propto B_{\varphi}(t) \propto$ $1 / s$. It follows from Eq. (6) that this condition is satisfied if $s \Omega^{\prime}=$ const. Then, $\nabla \times \boldsymbol{B}(t)=0$ and the Lorentz force is vanishing in Eq. (7). If, additionally, the centrifugal force is balanced by gravity, $g(s)=s \Omega^{2}$, then the pressure and hence, density are homogeneous in the basic state.

Consider stability of axisymmetric short wavelength perturbations with the spatial dependence $\alpha \exp (-\mathrm{i} \boldsymbol{k} \cdot \boldsymbol{r})$ where $\boldsymbol{k}$ is the wavevector. Small perturbations will be indicated by subscript 1 , while unperturbed quantities will have no subscript. For the purpose of illustration, we treat in this paper the simplest case when $\boldsymbol{k}$ has only the vertical component, $\boldsymbol{k}=(0,0, k)$. Then, the condition $\nabla \cdot \boldsymbol{B}_{1}=0$ yields $B_{1 z}=0$. The other non-trivial linearized MHD-equations to lowest order in $|\boldsymbol{k} \cdot \boldsymbol{r}|^{-1} \mathrm{read}$

$$
\begin{aligned}
& \frac{\mathrm{d} v_{1 z}}{\mathrm{~d} t}=\frac{\mathrm{i} k p_{1}}{\rho}+\frac{\mathrm{i}}{4 \pi \rho} k\left(\boldsymbol{B}_{1} \cdot \boldsymbol{B}\right), \\
& \frac{\mathrm{d} \rho_{1}}{\mathrm{~d} t}-\mathrm{i} \rho k v_{1 z}=0 \\
& \frac{\mathrm{d} B_{1 s}}{\mathrm{~d} t}=\mathrm{i} B_{s} k v_{1 z} \\
& \frac{\mathrm{d} B_{1 \varphi}}{\mathrm{d} t}=s \Omega^{\prime} B_{1 s}+\mathrm{i} k B_{\varphi}(t) v_{1 z} .
\end{aligned}
$$

Since gas is assumed to be isothermal, we have $p_{1} / p=\rho_{1} / \rho$. Eliminating all variables from Eqs. (8)-(11) in favour of the vertical velocity perturbation $v_{1 z}$ one obtains the following fourthorder ordinary differential equation in $v_{1 z}$ :

$\frac{\mathrm{d}^{4} v_{1 z}}{\mathrm{~d} t^{4}}+k^{2}\left[c_{s}^{2}+c_{\mathrm{A}}^{2}(t)\right] \frac{\mathrm{d}^{2} v_{1 z}}{\mathrm{~d} t^{2}}+6 \omega_{B \Omega}^{3} \frac{\mathrm{d} v_{1 z}}{\mathrm{~d} t}$
$+6 k^{2} c_{\mathrm{A} s}^{2}\left(s \Omega^{\prime}\right)^{2} v_{1 z}=0$,

where

$c_{\mathrm{A}}^{2}(t)=c_{\mathrm{A} s}^{2}+c_{\mathrm{A} \varphi}^{2}(t), \quad \boldsymbol{c}_{\mathrm{A} s}=\boldsymbol{B}_{s} / \sqrt{4 \pi \rho}$,

$\boldsymbol{c}_{\mathrm{A} \varphi}(t)=\boldsymbol{B}_{\varphi}(t) / \sqrt{4 \pi \rho}, \quad \omega_{B \Omega}^{3}=k^{2} c_{\mathrm{A} \varphi}(t) c_{\mathrm{A} s} s \Omega^{\prime}$.

Equation (12) with the corresponding initial conditions describes the behaviour of velocity perturbations on the time-dependent background state. The behaviour of perturbations of the radial and azimuthal magnetic field can then be calculated from Eqs. (10) and (11).

\section{Numerical results}

To follow the behaviour of perturbations, it is convenient to introduce the dimensionless quantities

$$
\begin{aligned}
& \tau=\Omega t, \quad q=\frac{s \Omega^{\prime}}{\Omega}, x=k H, H=\frac{c_{s}}{\Omega}, \\
& \beta_{s}=\frac{c_{\mathrm{A} s}^{2}}{c_{s}^{2}}, \quad \beta_{\varphi}=\frac{c_{\mathrm{A} \varphi 0}^{2}}{c_{s}^{2}}, \quad c_{\mathrm{A} \varphi 0}=\frac{B_{\varphi}(0)}{\sqrt{4 \pi \rho}} .
\end{aligned}
$$

Then, introducing $v=v_{1 z}(t) / v_{1 z}(0)$ where $v_{1 z}(0)$ is the initial velocity perturbation, we obtain from Eq. (12)

$$
\begin{aligned}
& \frac{\mathrm{d}^{4} v}{\mathrm{~d} \tau^{4}}+x^{2}\left[1+\beta_{s}+\left(\sqrt{\beta_{\varphi}}+q \sqrt{\beta_{s}} \tau\right)^{2}\right] \frac{\mathrm{d}^{2} v}{\mathrm{~d} \tau^{2}} \\
& +6 q x^{2} \sqrt{\beta_{s}}\left(\sqrt{\beta_{\varphi}}+q \sqrt{\beta_{s}} \tau\right) \frac{\mathrm{d} v}{\mathrm{~d} \tau}+6 q^{2} x^{2} \beta_{s} v=0 .
\end{aligned}
$$

The dependence of the solution on the wavelength is characterized by the parameter $x$ and, on the initial magnetization of gas, by the parameters $\beta_{s}$ and $\beta_{\varphi}$. To solve Eq. (13), one needs the initial conditions for three time derivatives of $v$. In actual calculations we try different initial conditions because their choice is determined by the origin of perturbations which is uncertain.

Equations (10), (11) can also be written in a dimensionless form. We have

$\frac{\mathrm{d} C_{\mathrm{A} s}}{\mathrm{~d} \tau}=\mathrm{i} x \sqrt{\beta_{s}} v(\tau)$

$\frac{\mathrm{d} C_{\mathrm{A} \varphi}}{\mathrm{d} \tau}=q C_{\mathrm{A} s}+\mathrm{i} x\left(\sqrt{\beta_{\varphi}}+q \sqrt{\beta_{s}} \tau\right) v(\tau)$,

where

$C_{\mathrm{A} s}=\frac{B_{1 s}(\tau)}{\sqrt{4 \pi \rho} v_{1 z}(0)}, \quad C_{\mathrm{A} \varphi}=\frac{B_{1 \varphi}(\tau)}{\sqrt{4 \pi \rho} v_{1 z}(0)}$.

In calculations, we assume that the initial perturbations of the magnetic field are vanishing. Equations (13)-(15) were solved numerically for a wide range of the parameters.

In Fig. 1, we plot the time dependence of $v, C_{\mathrm{A} s}$, and $C_{\mathrm{A} \varphi}$ in the regions where the angular velocity increases slowly with $s(q=0.1)$. The other parameters are $x=3, \beta_{s}=0.01$, and $\beta_{\varphi}=1$. Integrating Eq. (13), we choose the following initial conditions: $\mathrm{d} v(0) / \mathrm{d} \tau=1$ and $\mathrm{d}^{2} v(0) / \mathrm{d} \tau^{2}=\mathrm{d}^{3} v(0) / \mathrm{d} \tau^{3}=0$. 


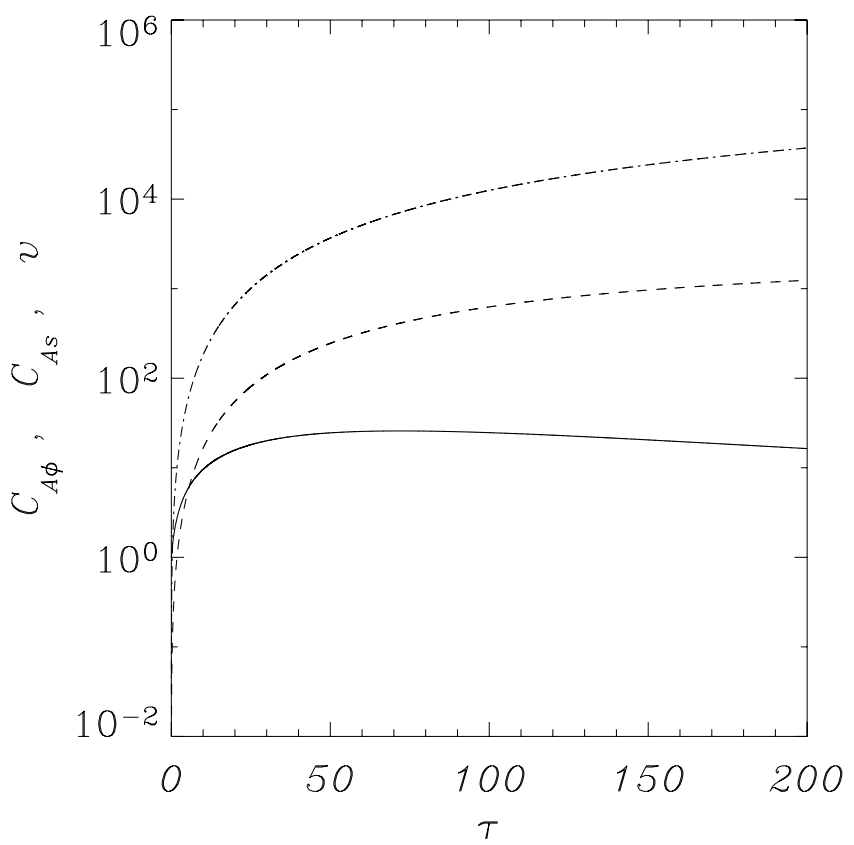

Fig. 1. The time dependence of $v$ (solid line), $C_{\mathrm{A} s}$ (dashed line), and $C_{\mathrm{A} \varphi}$ (dashed-and-dotted line) for $q=0.1, x=3, \beta_{s}=0.01$, and $\beta_{\varphi}=1$.

The behaviour of the velocity and magnetic field turns out to be substantially different. During the initial stage, the velocity reaches a flat maximum at $\tau \sim 60-70$ which corresponds approximately to $10 P$ where $P$ is the rotation period. At that time, the perturbation of velocity is $\approx 25$ times greater than its initial value. Perturbations of the magnetic field are much larger. After $\tau \sim 100(t \sim 15 P), C_{\mathrm{A} s}$ approaches the saturation level that is approximately a factor $10^{3}$ greater than the initial perturbation of $v_{1 z}$. The amplification of the azimuthal field is even larger. After $t \sim 15 P, C_{\mathrm{A} \varphi}$ is a factor $10^{4}$ stronger than the initial perturbation $v_{1 z}(0)$, and it continues to grow approximately linearly with time. Note that the long-term behaviour $(\tau \gg 200)$ of these quantities is also different. The velocity $v$ changes the sign and exhibits some sort of oscillatory behaviour with the amplitude growing $\propto t^{1 / 2}$ whereas $C_{\mathrm{A} s}$ reaches the saturation level. A perturbation of the azimuthal field $B_{1 \varphi}$ is still $\propto t$ at very large $t$.

In contrast with the magnetorotational instability, the behaviour of perturbations is not crucially sensitive to the sign of $\Omega^{\prime}$. To illustrate this point, we show in Fig. 2 the time dependence in the case $q=-0.1(\Omega$ decreases slowly with $s)$. Qualitatively, the dependences are the same. The only essential difference is that the azimuthal field generated by differential rotation has a negative sign because the radial gradient of the angular velocity is negative. Initially $(\tau<100)$, the main contribution to $B_{1 \varphi}$ is provided by compressibility (the second term on the r.h.s. of Eq. (11)). Later on, however the azimuthal magnetic perturbations are determined by winding up the radial field perturbations and becomes negative. In Fig. 2, the modulus of $C_{\mathrm{A} \varphi}$ is shown. Since $x$ is larger in this figure, perturbations of all quantities can reach higher values compared to Fig. 1. For instance, the velocity becomes $\sim 200$ times greater than the initial value after 15 rotation periods. The radial perturbation of the Alfvén velocity reaches the saturation level which is $\sim 2 \times 10^{4}$ times greater than $v_{1 z}(0)$, and the azimuthal perturbation is even about 10 times larger. For very long $t(\tau \gg 200)$, the velocity exhibits the oscillatory behaviour with the amplitude $\propto t^{1 / 2}$. In Fig. 3, we plot the behaviour of perturbations in the case of a very weak differential rotation, $q=0.01$. For a weak

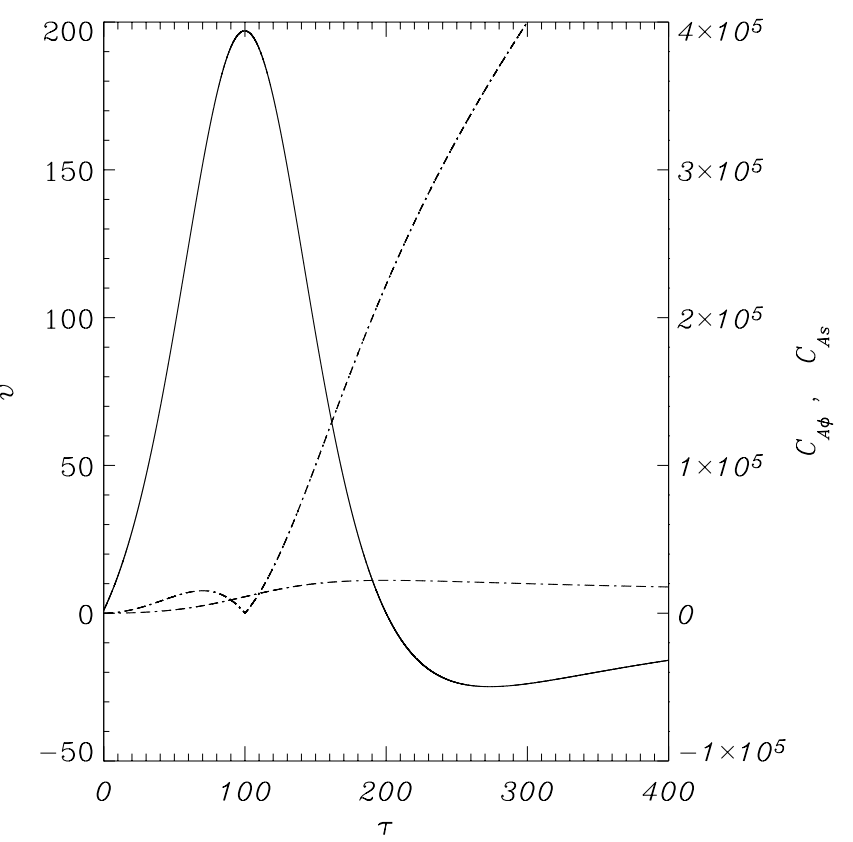

Fig. 2. Same as in Fig. 1 but for $q=-0.1$ and $x=10$.

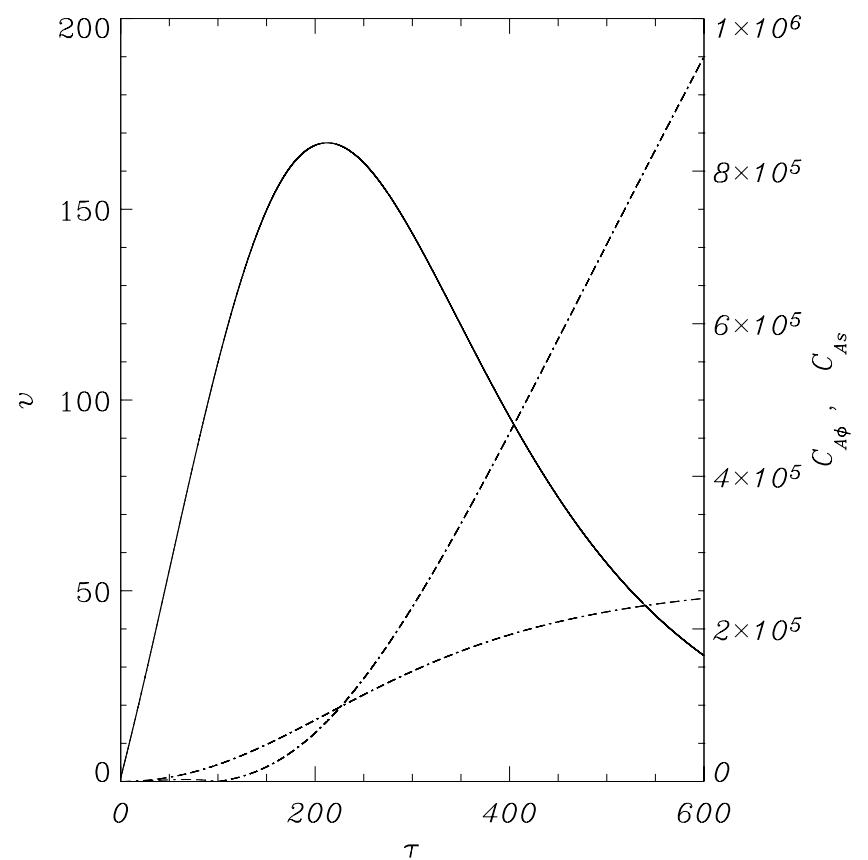

Fig. 3. Same as in Fig. 2 but for $q=-0.01, \beta_{s}=\beta_{\varphi}=0.1$, and $x=10$. The initial conditions are as for in Fig. 1.

rotation shear, the amplification of initial perturbations is slower. The velocity reaches the maximum after $\sim 40 P$, and this maximum is rather high: $v_{1 z}$ exceeds its initial value by a factor $\sim 100$. Despite a slower growth rate, saturation of $C_{\mathrm{A} s}$ still occurs at a rather high level $\left(C_{\mathrm{A} s} \sim 2 \times 10^{4}\right)$. As expected, the azimuthal field grows linearly with time and can reach a very high value.

The same dependences, for a relatively large value of $q$, are shown in Fig. 4. In this case, perturbations exhibit a clear oscillatory behaviour. This concerns particularly perturbations of the vertical velocity. After a short initial relaxation (lasting $\sim 2-3$ rotation period), $v$ reaches a self-similar regime when the amplitude of oscillations grows with time $\propto \tau^{1 / 2}$ and the frequency $\propto \tau$. Perturbations of the magnetic field also exhibit 


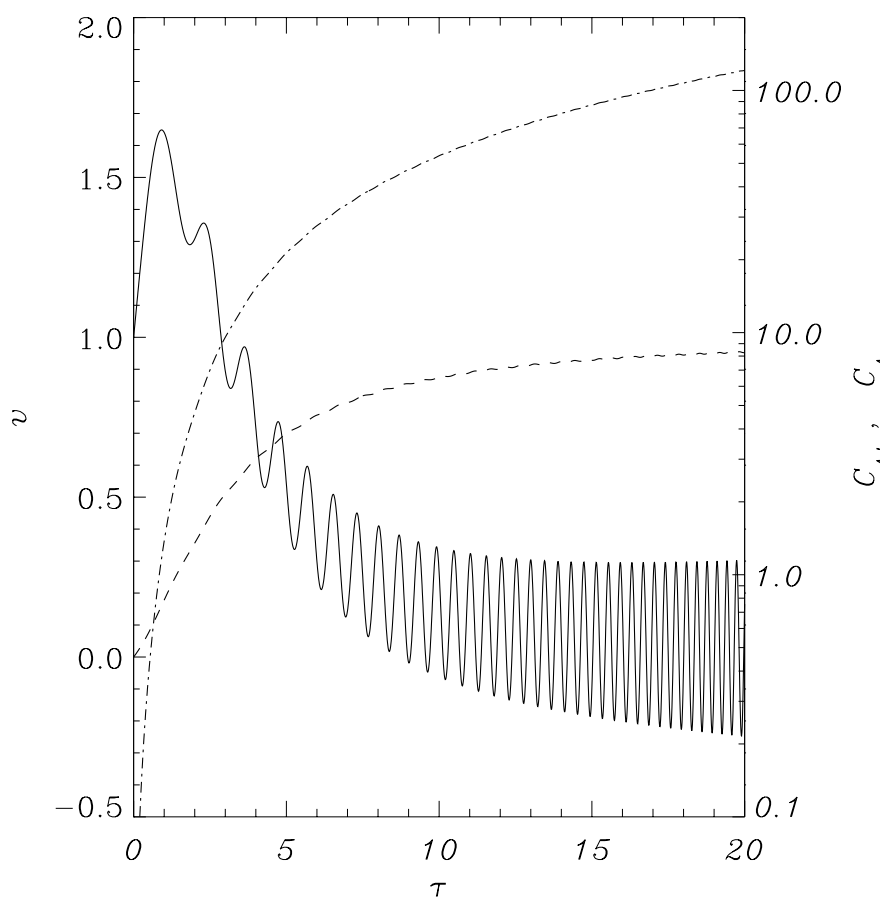

Fig. 4. Same as in Fig. 1 but for $q=1$ and $\beta_{s}=\beta_{\varphi}=0.1$.

a weak oscillatory behaviour but the amplitude of oscillations is much smaller. This is because the field components are given by the integrals of a rapidly oscillating function $v$ (see Eqs. (10) and (11)), and oscillations in $B_{1 s}$ and $B_{1 \varphi}$ are smoothed. Perturbations of the radial field reaches a saturation level after $\sim 2-3 P$. In saturation, the radial Alfvén velocity $C_{\mathrm{A} s}$ is approximately 10 times greater than the initial perturbation of the vertical velocity. Perturbations of the azimuthal field grow rather rapidly at the initial stage however, later on (at $\tau>2 P$ ), they continue to grow linearly with time.

In Fig. 5, we show the long-term behaviour of the amplitude of the vertical velocity. Except for the initial stage, the numerical results are well fitted by the same dependence $\propto \tau^{1 / 2}$ as that in Fig. 4. We can compare these numerical results with the analytical solution of Eq. (13) for large $\tau$. It can be easily checked that the asymptotic solution of Eq. (13) at large $\tau$ is given by

$v \propto \sqrt{\tau} \cos \left(\frac{1}{2} q x \sqrt{\beta_{s}} \tau^{2}+\frac{\pi}{8}\right)+O\left(\tau^{-3 / 2}\right)$.

Indeed, it can be noted that the vertical velocity is $\propto \tau^{1 / 2}$ and the frequency of oscillations is $\propto \tau$ at $\tau \rightarrow \infty$ in complete agreement with our numerical results. The frequency of oscillations depends on the magnetic field and is higher for a stronger initial radial field. Note that the asymptotic solution (17) is valid for a wide range of parameters and initial conditions. It is clear from solution (17) that $B_{1 s}$ should reach saturation quite rapidly. We have from Eq. (14) that

$C_{\mathrm{A} s}(\tau)=\mathrm{i} x \sqrt{\beta_{s}} \int_{0}^{\tau} v\left(\tau^{\prime}\right) \mathrm{d} \tau^{\prime}$.

The time integral of the asymptotic part of $v$ (Eq. (17)) gives a negligible contribution to $C_{\mathrm{A} s}$, and the only non-vanishing contribution can be provided by the value of $v$ at relatively short $\tau$. If $C_{\mathrm{A} s}$ reaches saturation rapidly then, as it follows from Eq. (15), $C_{\mathrm{A} \varphi}$ should grow approximately $\propto \tau$ at large $\tau$. This sort of behaviour agrees perfectly with the results of our numerical calculations.

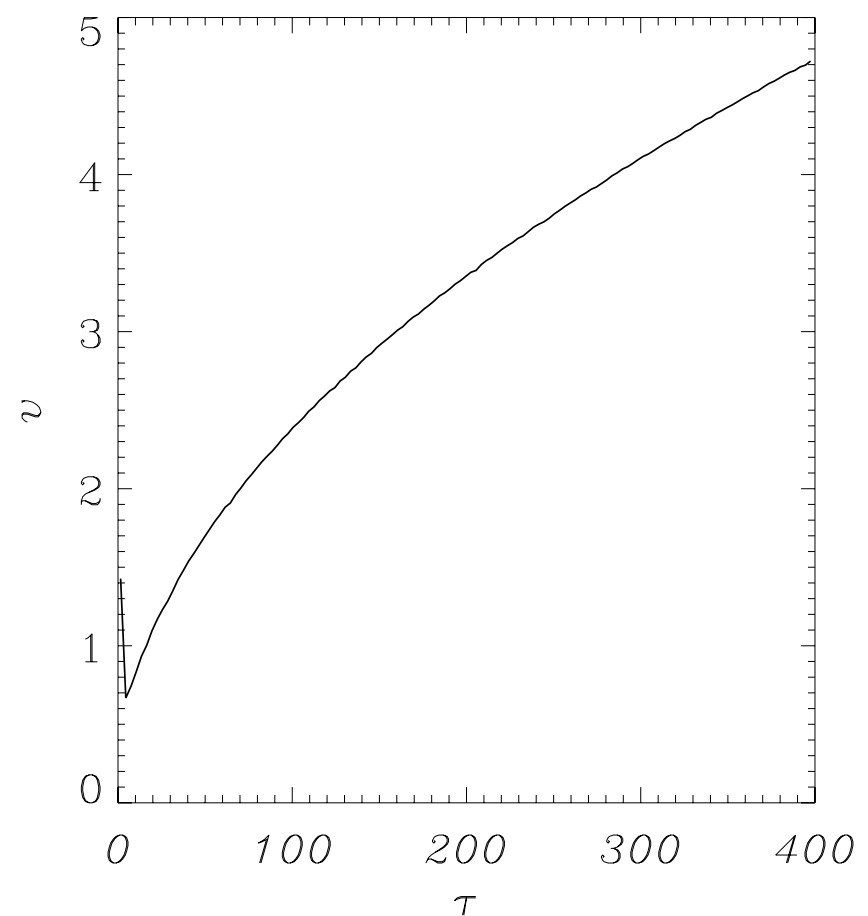

Fig. 5. The long-term behaviour of the amplitude of $v$ for $\beta_{s}=\beta_{\varphi}=0.1$, $q=2$, and $x=3$.

\section{Discussion}

We have shown that the winding up of toroidal field lines from the poloidal field lines is accompanied by an amplification of seed perturbations of the velocity and magnetic field. A very simplified model has been considered in this paper, but we believe that qualitatively the same results can be obtained for more general background states and perturbations. Differential rotation and compressibility of the gas lead to the generation of magnetosonic waves with the amplitude that grows with time. The physical processes responsible for this amplification are exactly the same that result in the instability considered by Bonanno \& Urpin $(2006,2007)$. The only difference is that, in this paper, we consider the development of perturbations on a time-dependent background state and, as a result, the growth of perturbations is not exponential.

The behaviour of seed perturbations depends essentially on various parameters and can generally be rather complex. If the parameter $q=s \Omega^{\prime} / \Omega$ is relatively small $(\leq 0.1)$, then the perturbations of velocity and magnetic field initially grow monotonously and can reach quite high values. For example, the perturbation of the vertical velocity becomes approximately 150-200 times greater than its initial value after only 15-30 rotation periods (see Figs. 2 and 3). Perturbations of the magnetic field reach even higher values during the initial stage. For instance, the Alfvén velocity corresponding to the perturbation of the radial field component, $C_{\mathrm{A} s}$, can exceed the initial velocity perturbation by a factor $\sim(1-3) \times 10^{5}$ after the same time, but the perturbations of the toroidal field are even stronger. As a result of such a strong initial amplification, seed perturbations can already reach a non-linear regime after 15-30 rotation periods if their initial values are sufficiently large. Further evolution of perturbations will then be entirely determined by nonlinear effects. However, if the non-linear regime is not reached 
during this initial stage, the behaviour of perturbations becomes oscillatory with slowly growing amplitude $\left(\propto t^{1 / 2}\right)$. At sufficiently large $t$, the frequency of oscillations grows linearly with time and is given approximately by

$\omega \approx \Omega\left(\frac{1}{2} k H \sqrt{\beta_{s}} s \Omega^{\prime} t\right)$.

Note that perturbations of the magnetic field exhibit more regular behaviour because they can be expressed in terms of the time integrals of a rapidly oscillating velocity. In the case of a strong differential rotation $(q \sim 1)$, perturbations exhibit the oscillatory behaviour from the very beginning and the initial growth of their amplitude is less significant.

The generation of magnetosonic waves occurs even if the magnetic field is very strong and suppresses different MHDinstabilities which can arise in a differentially rotating flow (for example, the MRI). The presence of differential rotation and radial magnetic field is, however, crucially important for the considered process. Since both differential rotation and radial field are quite common in astrophysics, we believe that the considered mechanism can occur in various astrophysical bodies and plays an important role in enhancing transport processes in plasma.
Acknowledgements. This research project has been supported by a Marie Curie Transfer of Knowledge Fellowship of the European Community's Sixth Framework Programme under contract number MTKD-CT-002995. V.U. thanks INAF-Ossevatorio Astrofisico di Catania for hospitality.

\section{References}

Acheson, D. 1978, Phil. Trans. R. Soc. London, 289A, 459

Agol, E., Krolik, J., Turner, N., \& Stone, J. 2001, ApJ, 558, 543

Balbus, S. A., \& Hawley, J. F. 1991, ApJ, 376, 214

Blokland, J. W. S., van der Swaluw, E., Keppens, R., \& Goedbloed, J. P. 2005, A\&A, 444, 337

Bonanno, A., \& Urpin, V. 2006, Phys. Rev. E, 73, 066301

Bonanno, A., \& Urpin, V. 2007, ApJ, 662, 851

Brandenburg, A., Nordlund, Å., Stein, R., \& Torkelsson, U. 1995, ApJ, 446, 741

Hawley, J. 2000, ApJ, 528, 462

Hawley, J., Gammie, C., \& Balbus, S. 1995, ApJ, 440, 742

Fricke, K. 1969, A\&A, 1, 388

Keppens, R., Casse, F., \& Goedbloed, J. 2002, ApJ, 569, L121

Kitchatinov, L. L., \& Rüdiger, G. 1997, MNRAS, 286, 757

Matsumoto, R., \& Tajima, T. 1995, ApJ, 445, 767

Narayan, R., Quataert, E., Igumenshchev, I., \& Abramowicz, M. 2002, ApJ, 577, 295

Pessah, M., \& Psaltis, D. 2005, ApJ, 628, 879

Urpin, V. 1996, MNRAS, 280, 149

Van der Swaluw, E., Blokland, J. W. S., \& Keppens, R. 2005, A\&A, 444, 347

Velikhov, E. P. 1959, Sov. Phys. JETP, 9, 995 